(NASA-CR-199525) FEMTOSECOND

N96-11954

PULSES GENERATED FROM A

SYNCHRONDUSLY PUMPED CHROMIUM-DOPED

FORSTERITE LASER (City Univ.. of

Unclas (NeW YOrK) $3 \mathrm{p}$

G3/36 0069843 


\title{
Femtosecond Pulses Generated from a Synchronously Pumped Chromium-Doped Forsterite Laser
}

\author{
A. Seas, V. Petritevic, and R. R. Alfano \\ Institute for Ultrafast Spectroscopy and Lasers, and \\ Center for Analysis of Structures and Interfaces \\ Departments of Physics and Electrical Engineering
}

The City College and Graduate School of the City University of New York

138 St. \& Convent Avenue, New York, N.Y. 10031, Tel. (212) 650-5531

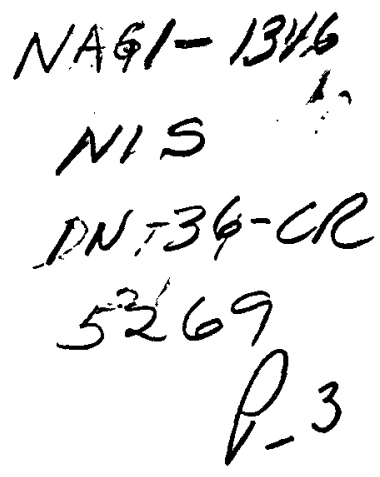

\section{Summary}

Kerr lens mode-locking (KLM) has become a standard method to produce femtosecond pulses from tunable solid state lasers. ${ }^{1}$ High power inside the laser resonator propagating through the laser.medium with nonlinear index of refraction, coupled with the stability conditions of the laser modes in the resonator result in a passive amplitude modulation which explains the mechanism for pulse shortening. Pulses of 17 fs FWHM were generated using KLM from Ti:Sapphire laser. ${ }^{2}$ Pulses of $50 \mathrm{fs}^{3}$ and pulses of $150 \mathrm{fs}^{4}$ were also generated from $\mathrm{Cr}^{3+}: \mathrm{LiSrSIF}_{6}$ using KLM in combination with a saturable absorber in the cavity and active mode locking, respectively. Recently, chromium doped forsterite was shown to exhibit similar pulse behavior. 5 Transform limited 60 fs pulses were generated from an actively mode locked forsterite laser using intracavity chirp compensation. The pulses were generated even when the active modulator was turned off, suggesting that KLM technique was operative in the generation of femtosecond pulses from $\mathrm{Cr}$ : forsterite laser.

In this presentation, we report on the first successful attempt to generate femtosecond pulses from a synchronously pumped chromium-doped forstenite laser with intracavity dispersion compensation. Stable, transform limited pulses with duration of $105 \mathrm{fs}$ were routinely generated, tunable between 1240 to $1270 \mathrm{~nm}$.

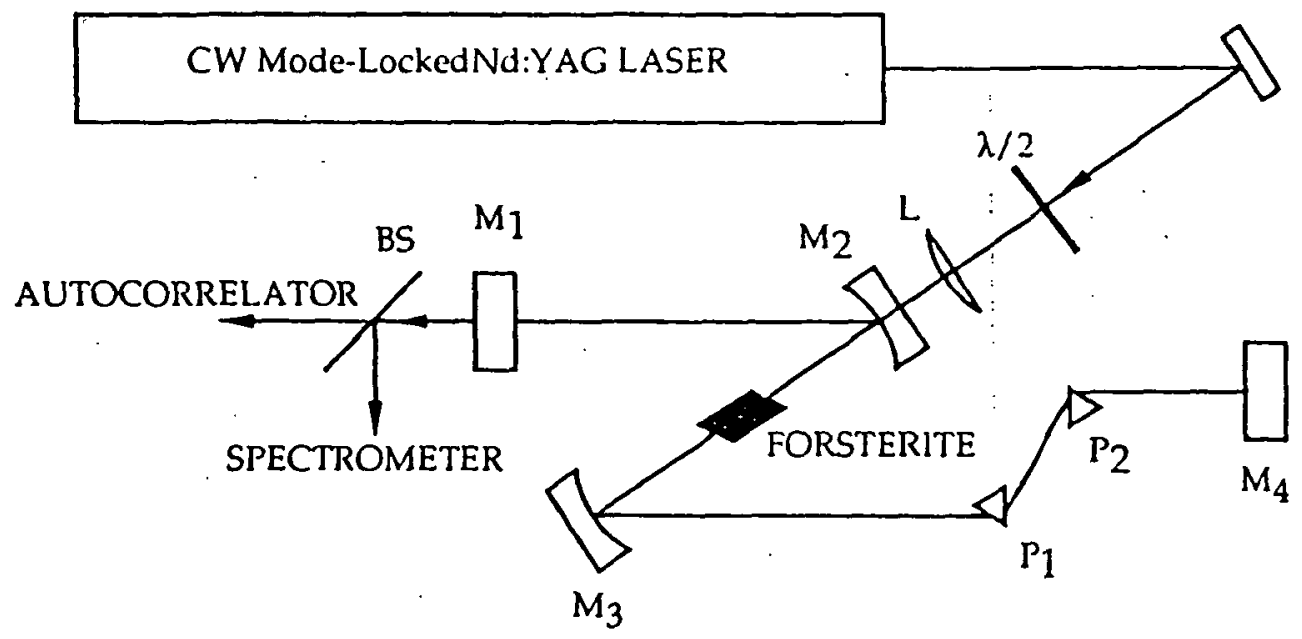

Fig. 1. Schematic diagram of the synchronously pumped mode-locked operation of the Cr.forsterite laser: $1 / 2$. half-wave plate for $1064 \mathrm{~nm} ; L$, focusing lens; $M_{1}$, output mirror, $M_{2}, M_{3}, 10$-cm-radius folding mirrors; $M_{4}$, back mirror; $B S$, beam splitter; $P_{1}$ and $P_{2}$, Scholt $S F 14$ glass prisms. 
The experimental arrangement is shown in Fig. 1. The Brewster-angle-cut forsterite crystal was placed in a four-mirror, $Z$-fold astigmatically compensated cavity. The combination of mirrors used was: a flat back mirror, two $10-\mathrm{cm}$-radius folding mirrors, and a flat output coupler. The transmission of the output coupler was $1 \%$ at the lasing wavelength, while the folding mirrors and the back mirror had reflectivity $\mathrm{R}=99.9 \%$ for the $1200-1300 \mathrm{~nm}$ range. The length of the sample was $1.2 \mathrm{~cm}$ with absorption coefficient at the pump wavelength of $1064 \mathrm{~nm}$ of $\alpha=1.1 \mathrm{~cm}^{-1}$. The laser crystal was mounted in a copper block and was cooled by a single-stage thermoelectric cooler. The Cr:forsterite crystal was pumped by an actively mode-locked Nd:YAG laser ( $82 \mathrm{MHz}$ ). The pump beam was focused by a $7.5-\mathrm{cm}$ lens through the $10-\mathrm{cm}$-radius folding mirror into the crystal. The 1064-nm pump power incident on the forsterite crystal was $5.3 \mathrm{~W}$, of which $3.9 \mathrm{~W}$ was absorbed by the crystal. The output of the forsterite laser was monitored with a fast germanium detector and an oscilloscope. The pulsewidth was measured with a noncollinear background free real-time intensity autocorrelator. A collinear interferometric autocorrelator was also used to determine the chirp characteristics of the output pulses. The bandwidth of the modelocked forsterite laser was measured using a $\mathrm{PbS}$ detector coupled to a $50-\mathrm{cm}$ Jarrel Ash monochromator, equipped with variable aperture slits.

Mode locking was observed when the length of the cavity was matched to the length of the mode locked Nd:YAG laser. Once mode-locking was achieved two regimes were observed. In the first we observe long duration pulses (200-300 ps FWHM). By further alignment of the cavity and slightly changing the position of the laser crystal and the distance between mirrors M2 and M 3 the pulsewidth was reduced down to $105 \mathrm{fs}$ with a spectral width of the order of $20 \mathrm{~nm}$. The output power of the forsterite laser when femtosecond pulses were generated was $45 \mathrm{~mW}$. An intensity autocorrelation trace and the corresponding spectrum of a typical pulse are shown in Fig. 2 (a) and (b). The pulsewidth shown is $105 \mathrm{fs}$ and the bandwidth is $16 \mathrm{~nm}$. The pulsewidth-bandwidth product $\Delta \tau_{p} \Delta v=0.32$, indicating transform-limited pulses for a sech ${ }^{2}$ pulse. Fig. 3 shows a picture of the interferometric autocorrelation trace of the output pulses. Since there is good visibility of the fringes at the winds of the pulse it is evident from this picture that the pair of prisms compensated for the nonlinear chirp introduced by the forsterite crystal. ${ }^{6}$
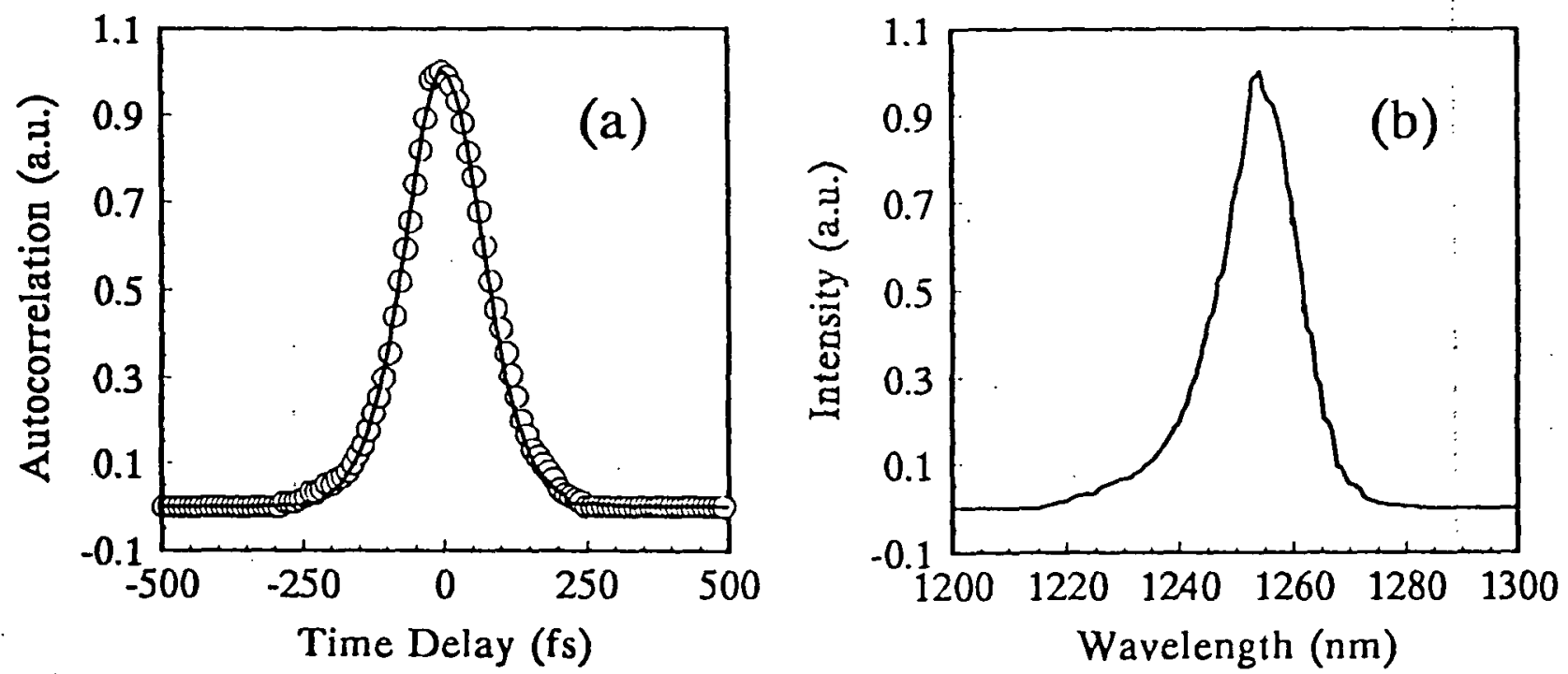

Fig. 2. An autocorrelation trace (a) and spectrum (b) of 105 - $\{$ s pulses obtainal from a $Z$ cavity with glass prisms for chirp compensation. Circles represent experimentil data and the solid line is the best fit. $\operatorname{sech}^{2}$ pulse shape was assumed for filting. The pulscwiduh-bandwidth product $\Delta \tau_{p} \Delta v=0.32$. 
The pair of prisms used in the cavity was made of SF14 Scholl optical glass. The optical characteristics of this type of glass for $1250 \mathrm{~nm}$ are $n=1.729, d n / d \lambda=-0.02017, d^{2} n / d \lambda^{2}=$ 0.02262 , and $\mathrm{d}^{3} \mathrm{n} / \mathrm{d}^{3}{ }^{3}=-0.0999 .7$ The optimum distance between the two prisms when stable 105-fs pulses were obtained, was determined to be $35 \mathrm{~cm}$. Carrying out the calculation for the phase derivatives with respect to frequency we get $\partial^{2} \phi / \partial \omega^{2}=-3273 \mathrm{fsec}^{2}$ and $\partial^{3} \phi / \partial \omega^{3}=-2170$ fsec $^{3} 8,9$ This indicates that SF14 is not the most appropriate glass for pulse shortening in this wavelength region since it introduces a large cubic phase term. This suggests that by using material which will introduce less cubic phase, shorted pulses will be generated as is the case with Ti:Sapphire. Different types of glass prisms will be discussed which minimize the effect of the cubic phase term.

The passively mode-locked forsterite laser was tuned using an aperture between prism $\mathrm{P}_{2}$ and mirror $\mathrm{M}_{4}$. We were able to continuously tune the laser between 1240 and $1270 \mathrm{~nm}$. The duration of pulses did not vary much throughout the whole tuning range.

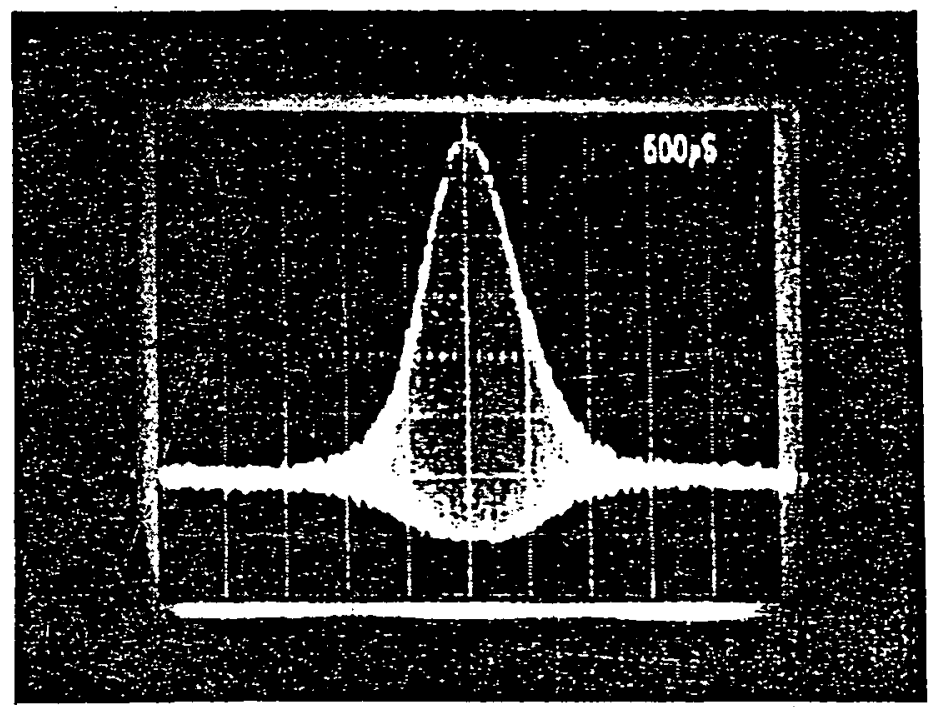

Fig. 4. Interferometric autocorrelation of the output pulses of the forsterite laser

In conclusion, transform-limited femtosecond pulses were generated from a synchronously pumped chromium-doped forsterite laser. Stable 105 femtosecond pulses in TEM 00 mode were obtained. The tuning range was of the order of $30 \mathrm{~nm}$, limited only by the coating of the mirrors used and the available pump power.

This research is supported in part by NSF, NASA and ARO.

\section{References}

1. T. Brabec, Ch. Spielmann, P. F Curley, and F. Krausz, Opt. Lett. 18, 1292 (1992), and references therein.

2. Chung-Po, Melanie T. Asaki, Sterling Backus, Margared M. Murnane, Henry C. Kapteyn, and Howard Nathel, Opt. Lett. 18, 1289 (1992).

3. N. H. Rizvi, P. M. W. French, and J. R. Taylor, Opt. Lett. 17, 877 (1992)

4. A. Miller, P. LiKamWa, B. H. T. Chai, and E. W. Van Stryland, Opt. Lett. 17, 195 (1992).

5. A. Seas, V. Petricević, and R. R. Alfano, Opt. Lett. 17, 937 (1992)

6. Jean-Claude M. Diels, Joel J. Fontaine, Ian C. McMichael, and Franscesco Simoni, Applied Optics 24, 1270 (1985)

7. "Optical Glass" Schott Catalog.

8. R. L. Fork, C. H. Brito Cruz, P. C. Becker, and C. V. Shank, Opt. Lett. 12, 483 (1987).

9. R. L. Fork, O. E. Martinez, and J. P. Gordon, Opt. Lett. 9, 1.50 (1984). 